\title{
Analysis on Design and Manufacturing Technology and Precision Machining Technology of Woodworking Machinery
}

\author{
Wang Bing ${ }^{1,2}$ \\ ${ }^{1}$ Xinxiang vocational and Technical College,Xinxiang, Henan, China \\ ${ }^{2}$ No. 6, jingsan Road, economic and Technological development zone, Xinxiang City, Henan Province
}

\section{Abstract:}

High speed machining (HSM) has become a development trend of modern manufacturing industry. It can not only significantly improve the production efficiency of products, but also greatly improve the machining accuracy and surface quality of production parts and reduce the production cost. Wood processing involves many processing processes such as sawing, milling and drilling. The compact four head motorized spindle can basically meet the milling and drilling requirements of wood processing. This paper studies the design, manufacturing technology and precision machining technology of woodworking machinery. This paper mainly adopts the finite element analysis method and applies the finite element analysis software ANSYS Workbench to simulate and model the woodworking four head high-speed motorized spindle, then analyzes and calculates its dynamic and static characteristics, and comprehensively considers the main influencing factors on the dynamic and static characteristics of motorized spindle. The test results show that the method proposed in this paper is of great significance to improve the actual working performance of motorized spindle.

Keywords: Wood, Woodworking Machinery, High Speed Machining, Static Characteristics.

\section{INTRODUCTION}

With the rise of the concept of "Green Industrial Revolution", the fourth industrial revolution, improving production efficiency has become an important goal of the development of industrial production technology [1-2]. High speed machining (HSM) can not only significantly improve the production efficiency of products, but also greatly improve the machining accuracy and surface quality of production parts, and reduce the production cost [3]. The core technology of high-speed machining mainly includes high-speed cutting principle, 
Article History: Received: 28 October 2021 Revised: 05 December 2021 Accepted: 10 January 2022 Publication: 28 February 2022

high-speed machining process, high-speed machining tool technology, high-speed machining machine tool technology and high-speed machining test technology [4-5]. Among them, highspeed machining machine tool technology is the material basis for realizing high-speed machining.

High speed machining machine tool technology mainly includes high-speed feed unit, highspeed motorized spindle unit, high-speed CNC control system, machine bed, cooling system, etc. Today, with the continuous development of modern manufacturing technology, the highspeed machine tool has inevitably become the development trend and trend [6-9].

The high-speed motorized spindle unit used in high-speed machine tools requires not only high working speed, but also low vibration, good machining stability and high machining precision under high-speed operation conditions. The static and dynamic characteristics of motorized spindle directly affect the precision of high-speed machining, machining surface quality, production efficiency, bearing capacity of machine tool and service life of bearing. Therefore, it is very necessary to analyze the dynamic and static characteristics of high-speed motorized spindle.

\section{DYNAMIC AND STATIC STIFFNESS ANALYSIS OF ANGULAR CONTACT BALL BEARING}

Angular contact ball bearing has many advantages, such as high limit speed, strong bearing capacity, high manufacturing precision, and can bear axial and radial loads at the same time. It is widely used in high-speed motorized spindle. Although the structure of angular contact ball bearing is simple, the actual stress state between its internal ball, cage and inner and outer rings is very complex, especially in the process of high-speed operation, the dynamic characteristics of the bearing become complex due to the action of gyro torque and centrifugal force, which affects the dynamic performance of the spindle unit. Therefore, the analysis of dynamic and static characteristics of diagonal contact ball bearing is an indispensable and important link to analyze the dynamic performance of spindle unit.

Hertz contact theory studies the stress-strain distribution of two objects after elastic deformation caused by compression contact. The basic assumptions are as follows:

(1) The contact object only produces elastic deformation, which obeys Hooke's law, that is, the stress-strain relationship is linear;

(2) Without considering the medium of the contact surface and the influence of friction, the load is vertically distributed on the surface of the contact surface;

(3) The contact surface is oval, approximate to quadratic paraboloid, and the size of the contact surface is much smaller than the curvature radius of the contact object surface.

Contact surface dimension of ball (long half shaft) a、 Short half shaft b), stress $\sigma(x, y)$ and 
Article History: Received: 28 October 2021 Revised: 05 December 2021 Accepted: 10 January 2022 Publication: 28 February 2022

deformation $\delta$ are [10]:

$$
\begin{array}{r}
a=\left(\frac{6 k^{2} \sum}{\pi E \sum \rho}\right)^{1 / 3} Q^{1 / 3} \\
b=\left(\frac{6 \sum}{\pi k E \sum \rho}\right)^{1 / 3} Q^{1 / 3} \\
\delta=r\left(\frac{9 \sum \rho}{2 \pi^{2} k^{2} E^{2} \sum}\right)^{1 / 3} Q^{2 / 3} \\
\sigma(x, y)=\sigma_{\max }\left[1-\left(\frac{x}{a}\right)^{2}-\left(\frac{y}{a}\right)^{2}\right]^{1 / 2}
\end{array}
$$

Where, $\mathrm{k}$ is ellipticity, $\mathrm{k}=\mathrm{a} / \mathrm{b}$; $\mathrm{E}$ is the elastic modulus; $\mathrm{E}$ is the contact load between the ball and the inner and outer rings; $r, \Sigma$ Three are complete elliptic integrals of the first and second kinds, respectively,

$$
\begin{aligned}
& r=\int_{0}^{\pi / 2}\left[1-\left(1-\frac{1}{k^{2}}\right) \sin ^{2} \varphi\right]^{-\frac{1}{2}} d \varphi \\
& \sum=\int_{0}^{\pi / 2}\left[1-\left(1-\frac{1}{k^{2}}\right) \sin ^{2} \varphi\right]^{\frac{1}{2}} d \varphi
\end{aligned}
$$

\section{FINITE ELEMENT ANALYSIS OF STATIC PERFORMANCE OF MOTORIZED SPINDLE}

The high-speed motorized spindle unit used in high-speed machine tools requires not only high working speed, but also low vibration, good machining stability and high machining precision under high-speed operation conditions. The static performance and dynamic characteristics of motorized spindle directly affect the precision of high-speed machining, machining surface quality, production efficiency, bearing capacity of machine tool and service life of bearing. Motorized spindle rarely fails due to fatigue fracture under actual working conditions. However, when the cutting force is too large or due to cutting self-excited vibration, the machining accuracy can not meet the production requirements. Therefore, in the design stage of motorized spindle, stiffness needs to be given priority relative to strength.

The static stiffness of the spindle unit reflects the ability of the spindle unit to resist static 
Article History: Received: 28 October 2021 Revised: 05 December 2021 Accepted: 10 January 2022 Publication: 28 February 2022

external load. The calculation formula is as follows:

$$
K=F / y(N / \mu m)
$$

The static stiffness of the spindle unit is generally divided into axial stiffness and radial stiffness. Generally, compared with the axial stiffness, the radial bending stiffness has a more significant impact on the machining accuracy. Therefore, the bending stiffness is used to refer to the spindle unit stiffness when measuring the spindle unit stiffness. The static stiffness of spindle unit is related to spindle overhang, support span, front and rear bearing stiffness, spindle material and structure.

In order to facilitate finite element analysis and calculation, it is necessary to simplify the spindle element model:

(1) The interference fit between the motor rotor and the spindle unit, the rotor and the shaft are equivalent to the same density material, which is equivalent to the spindle unit model as a concentrated mass;

(2) The bearing is simplified as elastic support, only radial stiffness is considered, and the influence of axial stiffness and angular stiffness is ignored;

(3) Ignoring the change of stiffness when the bearing is under pressure, the bearing stiffness is regarded as a fixed value.

The calculation formula of static stiffness $\mathrm{K}$ of spindle unit is:

$$
K=\frac{F}{y}
$$

The static stiffness $\mathrm{K}$ of the spindle unit is:

$$
K=\frac{F}{y}=\left[\frac{a^{3}}{3 E I}\left(\frac{l}{a}+1\right)+\frac{(a / l)^{2}}{K_{A}}+\frac{(1+a / l)^{2}}{K_{B}}\right]^{-1}
$$

According to formula (9), the static stiffness of spindle unit is related to spindle suspension, support span, front and rear bearing stiffness, spindle material and structure. Among them, the stiffness of spindle unit increases with the reduction of spindle overhang. Therefore, when designing spindle, the overhang should be reduced as far as possible if the structure allows; The higher the stiffness of the front and rear bearings, the greater the stiffness of the spindle unit. Therefore, selecting appropriate bearings can improve the static performance of the spindle, such as Si3N4 mixed ceramic ball bearing with dense beads; The influence of support span on the stiffness of spindle unit is complex. In addition, the spindle is generally a stepped shaft and the end is equipped with tool taper hole, so it is difficult to obtain a reasonable support span from the formula; In addition, reasonable selection of spindle material and optimization of spindle structure can improve the stiffness of spindle unit.

One set of DB is equipped with angular contact ball bearings, and its preload is estimated to 
Article History: Received: 28 October 2021 Revised: 05 December 2021 Accepted: 10 January 2022 Publication: 28 February 2022

be = rated dynamic load $/ 15 \mathrm{kN}$ (medium load). It can be obtained that the single stiffness of the front bearing is $3826 \mathrm{~N} / \mu \mathrm{m}$, and the angular contact ball bearing DB configuration is approximately equal to the superposition of two bearings, so the stiffness value of the front bearing is $7652 \mathrm{~N} / \mu \mathrm{m}$. The approximate formula is adopted for the rear bearing, and the calculated preload is $200 \mathrm{~N}$, and the stiffness value of the rear bearing is $1316 \mathrm{~N} / \mu \mathrm{m}$.

TABLE I. Bearing parameters of $2.2 \mathrm{~kW}$ spindle unit

\begin{tabular}{|c|c|c|}
\hline BEARING PARAMETERS & FRONT BEARING & REAR BEARING \\
\hline MODEL & 71906C/HQ1DB & $6206 \mathrm{C}$ \\
\hline INNER DIAMETER / MM & 30 & 30 \\
\hline OUTER DIAMETER / MM & 47 & 62 \\
\hline CONTACT ANGLE & 15 & 15 \\
\hline NUMBER OF SCROLLS & 24 & 16 \\
\hline ROLLING ELEMENT \\
DIAMETER / MM & 5 & 8 \\
\hline RATED DYNAMIC LOAD / KN & 7.6 & 11.5 \\
\hline RATED STATIC LOAD / KN & 7.2 & . \\
\hline \multirow{2}{*}{ LIMIT SPEED / R $\cdot$ MIN $^{-1}$} & 26000 grease lubrication & 9500 grease lubrication \\
\cline { 2 - 3 } & 40000 oil lubrication & 13000 oil lubrication \\
\hline
\end{tabular}

It is concluded that the static stiffness $\mathrm{K}$ of $2.2 \mathrm{~kW}$ spindle unit is:

$$
K=\frac{F}{y}=5000 \mathrm{~N} / 56.702 \mu \mathrm{m}=88.180 \mathrm{~N} / \mu \mathrm{m}
$$

This motorized spindle is used for wood processing, and its static stiffness meets the design requirements of woodworking four head high-speed motorized spindle. At present, there is no specific verification standard for the stiffness of spindle unit or bearing. The greater the stiffness, the higher the machining accuracy, but the flexibility is conducive to eliminate the flux instability caused by the gap between stator and rotor. In order to eliminate the influence of eccentric torque, the static stiffness of motorized spindle can be verified by reference to the design standard.

\section{FINITE ELEMENT ANALYSIS OF MODE OF MOTORIZED SPINDLE}

The natural frequency of motorized spindle structure and the corresponding modal structure shape are important parameters for designing the structure under varying load conditions. Modal analysis is used to calculate the natural frequency and modal shape of the structure. 
Article History: Received: 28 October 2021 Revised: 05 December 2021 Accepted: 10 January 2022 Publication: 28 February 2022

Modal analysis studies the free vibration of undamped system and determines the vibration characteristics of spindle unit. That is, natural frequency and vibration mode are the basis of other dynamic analysis (such as harmonic response analysis, response spectrum analysis and transient dynamic analysis).

According to the structural characteristics of the spindle unit, its mathematical modeling is carried out, and the following assumptions are made to simplify the solution:

(1) In the angular contact ball bearing, the Elastohydrodynamic lubricating oil film between the ball and the ring raceway is very thin, so the influence of the oil film in the bearing and the damping in the spindle unit is ignored;

(2) The spindle unit will not have torsional vibration, and the support is an axisymmetric structure, so the influence of bearing angular stiffness is ignored and only transverse bending vibration in one direction is considered;

(3) The interference fit motor rotor is loaded on the spindle unit as an additional mass, ignoring the influence of the bending stiffness and temperature field effect of the additional parts.

(4) The influence of temperature on the dynamic performance of spindle unit is ignored.

For the eigenvalue extraction of modal analysis, ANSYS software provides a variety of methods: block Lanczos method, subspace method, power dynamics method, reduction method, asymmetry method, damping method and QR damping method. Compared with other methods, block Lanczos method has the advantages of high precision and fast calculation speed. Therefore, block Lanczos method is selected to extract the eigenvalues of motorized spindle modal analysis in this paper.

The differential equation of motion of the spindle element of any order viscous damping system is:

$$
M \ddot{X}(t)+C \dot{X}(t)+K X(t)=F(t)
$$

Among them,

$$
K=K_{S}+K_{B}
$$

Where, $\mathrm{K}_{\mathrm{S}}$ is the spindle stiffness matrix and $\mathrm{K}_{\mathrm{B}}$ is the bearing stiffness matrix.

The damping coefficient of rolling bearing is relatively small, which has little effect on the calculation of the natural frequency of spindle unit, and can be ignored. When the external excitation force is not considered, the undamped free vibration differential equation of the spindle unit is:

$$
M \ddot{X}(t)+K X(t)=0
$$

Suppose the form of the solution of equation (13) is;

$$
x_{i}+A_{i} \sin \left(\omega_{i} t+\varphi\right)(i=1,2, \ldots, n)
$$


Article History: Received: 28 October 2021 Revised: 05 December 2021 Accepted: 10 January 2022 Publication: 28 February 2022

Bring equation (14) into equation (13) to obtain:

$$
K A-\omega^{2} M A=O(15)
$$

Where, $\mathrm{w}_{\mathrm{i}}$ is the angular frequency; $\mathrm{A}$ is the main vibration mode corresponding to $\mathrm{w}_{\mathrm{i}}$.

\section{CONCLUSION}

In this paper, the woodworking four head high-speed motorized spindle is taken as the research object. According to the structure of motorized spindle and the characteristics of dynamic and static characteristics of spindle unit, combined with the analysis of internal dynamic state and dynamic stiffness of spindle bearing at high speed, the static and dynamic characteristics of motorized spindle are studied respectively. Through this study, we can draw the following conclusions:

(1) Based on the characteristics of wood processing, the motorized spindle adopts the form of motor central spindle, and the shaft end seal adopts the form of combination of sealing ring and labyrinth seal. Angular contact mixed ceramic ball bearing and deep groove ball bearing are selected as the bearings. The paired bearings are in $\mathrm{dB}$ (back-to-back) configuration for positioning and preloading. The spring of single deep groove ball bearing is preloaded under constant pressure, the motor cooling system adopts the form of compressed air cooling, and the dynamic balance adopts the weight removal method. The overall structure of motorized spindle is compact, and all performance parameters meet the design requirements.

(2) Based on Hertz contact theory, the force and deformation of angular contact ball bearing under static load are analyzed, and the radial stiffness of bearing under static load is deduced, which provides the theoretical stiffness value of bearing boundary constraint for the static performance analysis of motorized spindle. The finite element method is used to analyze the dynamic stiffness of the bearing, which avoids the nonlinear characteristics of the dynamic characteristic analysis of the spindle element, and obtains a reasonable bearing stiffness value to meet the requirements of the dynamic analysis of the spindle element.

\section{REFERENCES}

1. Wang Fujie, Shuang Yuanhua, Hu Jianhua. Design and Analysis of Locking Mechanism of Piercing Top Bar Trolley. Manufacturing Automation, 2013, 16: 125-126130

2. Tang Zhigang, Cui Guogang, Fan Zhimin. Design and Analysis of Suspension Cushion of Truck Cab Locking Mechanism Assembly. Electromechanical Engineering, 2017, 34 (005): 470-473

3. Zhao Fanggeng, Jiang Ding, Zhu Xianmin. Study on Control Strategy of Automatic Control System for Differential Locking Mechanism of Off-road Vehicle. Automotive Engineering, 2005 (01): 99101 
Article History: Received: 28 October 2021 Revised: 05 December 2021 Accepted: 10 January 2022 Publication: 28 February 2022

4. E Ruidi, Zhao Ziqiang, Li Dangsheng. Research on Motion Interference and Profile Modification of Internal Gear Disk Block Locking Mechanism. Mechanical Design and Manufacturing, 2018 (7): 6369

5. Tian Guangcai, Gao Bo, Yang Zhen. Strength Analysis of Differential Locking Mechanism Sleeve. Mechanical Design and Manufacturing, 2010, 10: 181-183

6. Zhao Xiaoyu, Wang Xiaocong, Wang Yansong. Study on Locking Time of Composite Sensitive Safety Belt. China Mechanical Engineering, 2015, V.26; No.432 (24): 103-107

7. Wu Zhaocheng, Han Guangxin. Optimization Design of the Hinge Point Position of Steering Lock Rod for Multi Axle Steering Drive Axle. Automotive Technology, 2003, 2: 5-8

8. Zhu Xianmin, Gao Bo, Jiang Ding. Analysis of the Strength and Motion of the Jaw Clutch in the Automatic Control System of the Differential Synchronous Locking Mechanism of Off-road Vehicles. Automotive Technology, 2001, 1 (2): 10-13

9. Zhao Fanggeng, Jiang Ding, Zhu Xianmin. Design of Ecu for Automatic Control System of Differential Locking Mechanism of off Road Vehicle. Automotive Engineering, 2003, 25 (005): 501505

10. Gao Bo, Jia Jumin, Qiao Yongwei. Design of Gear Profile of Synchronizer for Differential Locking Mechanism of off Road Vehicle. Mechanical Science and Technology, 2002 (05): 777-779 